\title{
The impact of asymmetry on aortic root measurements in patients
} with bicuspid aortic valves

\author{
Felipe S Torres ${ }^{*}{ }^{1}$, Andrew M Crean ${ }^{1}$, Elsie T Nguyen ${ }^{1}$, Narinder Paul ${ }^{1}$, \\ Timothy Bradley ${ }^{2}$, Jack Colman ${ }^{1}$ and Rachel M Wald ${ }^{1}$
}

\begin{abstract}
Address: ${ }^{1}$ Toronto General Hospital, Toronto, ON, Canada and ${ }^{2}$ Hospital for Sick Children, Toronto, ON, Canada
\end{abstract}
* Corresponding author

from 13th Annual SCMR Scientific Sessions

Phoenix, AZ, USA. 21-24 January 2010

Published: 21 January 2010

Journal of Cardiovascular Magnetic Resonance 20 I0, I2(SuppI I):PI39 doi:I0.I I86/I532-429X-I2-SI-PI39

This abstract is available from: http://jcmr-online.com/content/I2/SI/PI39

(C) 2010 Torres et al; licensee BioMed Central Ltd.

\section{Introduction}

Bicuspid aortic valve (BAV) is the most common form of congenital heart disease. Current guidelines to direct surgical intervention are derived from echocardiographic measurements. Measurements of the aortic root using cardiovascular magnetic resonance imaging (CMR) in this population have not been well studied but may allow better characterization of aortic root morphology.

\section{Purpose}

We sought to evaluate CMR derived aortic root measures and to correlate these with standard echocardiographic measures as obtained by $2 \mathrm{D}$ transthoracic echocardiography (TTE).

\section{Methods}

Steady-state free precession cine acquisitions across the aortic root in short axis in patients with a diagnosis of BAV from January 2006 to December 2008 were evaluated at our institution using a 1.5 T magnet. For each aortic root, end-systolic measurements were obtained in duplicate by a single observer from cusp-to-commissure (3 measurements: right, left and non-coronary cusps to opposite commissure respectively) and cusp-to-cusp (3 measurements: right-left, right-non and left-non coronary cusps) using CMR. Using TTE, measures of the aortic root were recorded from the parasternal long axis view. Aortic root asymmetry was defined by comparing the standard deviation (SD) of the average of the 3 measurements of an individual $(\mathrm{SDi})$ to the $\mathrm{SD}$ of the entire population $(\mathrm{SDp})$.
Two groups were generated: symmetric (SDi < = SDp) and asymmetric (SDi $>\mathrm{SDp}$ ) aortic roots.

\section{Results}

A total of 55 consecutive patients (37 males [67.3\%], mean age $32.4 \pm 10.3$ years) were included. The intrareader reproducibility of each of the $6 \mathrm{CMR}$ measurements was excellent (intra-class correlation coefficient > = 0.996 for all measures). For those with symmetric aortic roots, Pearson correlation coefficient for cusp-cusp measures by CMR as compared to TTE was $r=0.948$ and for cusp-commissure measures was $r=0.952$. For those with asymmetric aortic roots, Pearson correlation coefficient for cusp-cusp measures by CMR as compared to TTE was $r$ $=0.798$ and for cusp-commissure measures was $r=0.804$. There was a statistically significant difference between the correlation coefficients between the symmetric and asymmetric groups for cusp-cusp measurements and near statistical significance for the cusp-commissure measurements for CMR as compared to TTE ( $p=0.029$ and $\mathrm{p}=0.056$, respectively).

\section{Conclusion}

Although there are good correlations between CMR and TTE derived measurements of the aortic root in patients with BAV, aortic root asymmetry decreases the strength of the correlation between CMR and TTE measurements. When there is aortic root asymmetry, cusp-commissure measurements obtained by CMR are better correlated with TTE measures as compared with cusp-cusp measurements. 